\title{
Tissue Microarrays as a Tool in the Discovery and Validation of Tumor Markers
}

\author{
Stephen M. Hewitt \\ Tissue Array Research Program, Laboratory of Pathology, Center for Cancer Research, National \\ Cancer Institute, National Institutes of Health, Bethesda, MD, USA.
}

\section{Summary}

Tissue microarrays are a platform of condensed histopathology that has revolutionized the translation of basic science to clinical utility. Tissue microarrays have resulted in a paradigm shift from histopathology to immunopathology and moved analysis of small selected samples sets of tens of specimens to a high-throughput environment of hundreds of specimens. Tissue microarrays have influenced validation strategies, but have a role in discovery as well, allowing a pathways approach to analysis of tumors.

\section{Keywords}

Tissue microarray; Immunohistochemistry; Pathology; Histology; Tumor; Formalin-fixed paraffinembedded tissue

\section{Introduction}

Identification of tumor markers is but one step of the process to improved patient outcomes in cancer. There are a plethora of tools and models for the identification of tumor markers. The discovery process for a tumor marker is a complex multistep process. An essential step in the process is generalization of any finding to a larger set of samples, ultimately within the context of the disease process (cancer).

With cancer, ultimately a tumor marker is related to the tumor - either the marker is derived from the tumor cells themselves or is in response to the tumor's presence. Cancer is a disease of proliferative cells, and hence the examination of tissue, via histopathology, is a key element of diagnosis, prognosis, and prediction of response to therapy. We have the inherent advantage of being able to visualize, with the aid of a microscope, the cancer cells, and distinguish how they are different for the normal tissue. Traditionally this has been purely based on histomorphology, the appearance of the cells at the light microscope level after the application of a simple stain, such as an H\&E.

The tissue microarray (TMA) has revolutionized this process. The process remains cell based; however, the shift is now from histomorphology to presence of specific proteins as 
detected most commonly by imrnunohistochemistry. A TMA is a relatively simple construct of cores of tissue extracted from tissue blocks and reinserted into a recipient block (1). This TMA block is then sectioned to yield a TMA slide which can then be subject to any analysis that is routinely applied to tissue. The key elements are that the selection of the tissue removed is directed and that its placement in the recipient is directed so that the array is an organized structure.

\section{What Can Be Placed in a TMA?}

Virtually any biologic specimen can be arrayed. Although rare, TMAs can be constructed of plant material and other biologic specimens. The majority of TMAs are constructed of archival human tissue obtained from departments of pathology (2). This is the same tissue that is removed for therapeutic and diagnostic considerations in the process of medical care. Autopsy tissue is routinely encountered; however, the rate of autopsy in the US has declined significantly, not to mention issues of tissue quality with prolonged postmortem times (socalled warm ischemia times), making this tissue less useful and less plentiful than many researchers believe.

Use of human tissue requires appropriate ethical review and safeguards. The guidelines and rules for use of human diagnostic pathology material vary greatly at the local, state, and national levels. The subject is too complex to review here, but if you are constructing a TMA, it is imperative to obtain appropriate approvals and implement safeguards on patient identities. If you are obtaining a TMA, you must ensure you follow the rules and regulations of your institution, in addition to those of the source institution.

Just because a tumor has a described natural history, does not mean that samples of the tumor are available in the archives of your local pathology department. With the advances in diagnostic modalities, especially radiology, not to mention the advances of treatment for cancer beyond surgery (radio- and chemotherapy), many tumors, especially metastatic tumors are rarely resected, or even biopsied, and as a result are not available for inclusion in TMAs. For those interested in construction of progressionrelated TMA; multi-institutional studies, potentially with tissue procurement protocols, should be considered (3).

Although human tumors are the most common source of tissue for TMAs, other tumor models systems are routinely encountered. Mouse tissue, is routinely utilized for the construction of TMAs, either consisting of tumors from transgenic lines, or other rodent tumor model systems. Dogs are routinely utilized as a model for some human cancers, including osteosarcoma and lymphoma. Typically these are companion animals, pets in the community, and their tissue is obtained in the same means as human tissue - from diagnostic and therapeutic interventions (4).

Beyond tumor and normal tissue, the most commonly arrayed specimens are cell lines and xenografts. Cell line arrays (CMAs) are routinely constructed from panels of cell lines either as stand-alone platforms for research or inclusion as controls in other TMAs. The NCI60, a panel of 60 cell lines used by the NCI for screening of chemotherapeutic agents, has been 
arrayed (2). As an extension of this, xenograft arrays (XMAs) can be constructed from tumors transplanted into mice $(2,5)$.

\section{What Is Appropriate Material for a TMA?}

TMAs are routinely constructed of formalin-fixed, paraffin-embedded tissue $(1,6)$. Frozen tissue can be utilized (7); however, it is an extremely delicate resource, and the challenges of constructing a TMA of frozen tissue, let alone its storage, generally prevent the construction of frozen-tissue-based TMA. After reading this chapter, it should be apparent when a frozen tissue TMA is appropriate, and when not.

Alternative fixatives for paraffin-embedded tissues are routinely encountered both in research and clinical care environments. Ethanol as a fixative, typically employed as a $70 \%$ solution, in place of $10 \%$ neutral buffered formalin is an excellent choice for some studies (8). Other fixatives have been utilized; however, they tend to result in hard tissues, which, especially in older archival material, tend to crumble and produce suboptimal arrays. Bouin's fixative is an acid-based fixative and should be avoided on this account (6). Decalcification is a common issue. There are two common methods of decalcification: acid and EDTA. Neither are perfect and both are documented to damage some antigens; however; EDTA seems to be preferred in the construction of TMAs (6).

Regardless of the choice of fixative, it is important, at a minimum to know which tissue samples are fixed in what fixative; otherwise, appropriate staining conditions for immunohistochemistry are illusive. Differences in tissue handling and processing can impart enormous impacts on inununohistochemical assays, and these problems become magnified on TMAs (9). Often there is little a researcher can do but be aware of these pitfalls. If possible, it is best to avoid mixing tissues with different types of fixation on a TMA. If this is not possible, grouping them by fixative may assist in debugging assays and interpretation.

\section{What Assays Can Be Applied to a TMA?}

If you can perform the assay on a piece of tissue on a microscope slide, you can probably apply it to a TMA. The vast majority of assays on TMAs are immunohistochemical stains. Routine histochemical stains, most commonly an H\&E stain, are frequently applied.

In situ assays, either for RNA or DNA, visualized either by radioactivity, fluorescence, ECL, or chromagenic means, can be applied to a TMA (10-12). In situ assays are less frequently employed as an application largely because the complexity of assay renders them rare in general, replaced by PCR, as well as the complexity of interpretation. Interpretation of in situ assays is frequently performed at high magnification, and the high density of TMAs makes data collection tedious in the absence of sophisticated imaging systems. Although DNA quality is rarely a problem, RNA quality in formalinfixed tissue can be a limiting issue for low abundance transcripts; however, the literature does document detection and quantification of such transcripts by in situ hybridization $(10,11)$.

Other assays, including Fourier transform infrared spectroscopy and Raman spectroscopy, have been applied to TMAs (13). These are examples where the TMA platform has truly 
enabled the technology by providing the high-throughput platform required for analysis. Two assays that in general should not be interpreted on TMAs are mitotic counts and vessel density assays for angiogenesis (14). Both of these assays depend on determining an average number of events per fields observed, and the core sizes are inadequate of an appropriate sampling.

\section{Adequacy of Number and Size}

One of the most common questions asked of those who work wdth TMAs routinely is how do you determine how many samples and what core size to construct the TMA. The simplest answer is "more is better." In general a larger number of samples is more important than larger cores. We typically do not construct TMAs of less than 25 samples and from a statistical point of view, for determining a difference between two groups, 50 total samples dhaded equally two groups is optimal. The TARP lab does not place more than 500 cores of 0.6-mm diameter on a single section for technical reasons in manufacture, assay performance, and interpretation. Rarely does an investigator have more than 500 samples, and as the number of samples dwindles, the core size should increase. With the advent of new arrayers and a larger community or researchers, guidelines are becoming more difficult to reach consensus on.

Arguments of adequate tissue sample size are common. The simple fact is that tissue always has some level of heterogeneity, and different cells are of different sizes, not to mention admixture with normal cells, blood vessels, and stroma. For any marker, the adequacy of sampling is independent and can only be determined by experimentation and comparison to whole sections, for which there is a reasonable outcome measure, most commonly survival. As a rule of thumb, a $0.6-\mathrm{mm}$ core to tissue contains $\sim 1,500$ epithelial cancer cells. If $5 \%$ of the cells express the given marker, that is 75 positive cells. For reference, many CMAs are constructed to contain between 100 and 300 cells. There are two exceptions that require mention. TMAs of osteosarcoma are best constructed with $1.0-\mathrm{mm}$ needles as the needle wall thickness is optimal for strength and the cores are not so large as to be excessively cracked. CMAs are frequently constructed with only $6-\mathrm{mm}$ or $1.0-\mathrm{mm}$ needles as the cores offer no histomorphology and are limited to cytomorphology, requiring a minimum of 200x total magnification.

\section{Why Would I Need a TMA?}

All roads lead to a TMA, or so it would seem in some instances. In fact, when working with tumor markers, all roads lead to testing in patient samples and most commonly today, before a tumor marker is tested in patient samples it is screened on a TMA (2). The genesis of TMAs was to screen tumor markers against human samples (1) and in general this remains the main use; however, how an investigator comes to interrogate a TMA has changed over time.

The schema of arriving at a TMA experiment can be described in two ways mechanistically - what experiments lead you to a TMA, or theoretically, what is the utility of a TMA The theoretic will be discussed first, followed by the common mechanistic examples. 
Tumor markers are generally broken into three groups: diagnostic, prognostic, and predictive (15). Diagnostic markers are those that render a finding of the presence or type of disease. This was anticipated to be significant role for TMAs - identification of new tumor markers; however, there is limited need for new diagnostic markers. Cancer vs. benign is frequently easily distinguished, and few protein biomarkers have utility discriminating cancer vs. benign. There remain questions of tumor origin (lineage), and TMAs have demonstrated utility (16) in development of these markers but it is not a common use.

Prognostic markers are those that predict the natural behavior of a tumor. In clinical care, grade and stage of a malignancy are used to prognosticate the behavior of a tumor and guide the decision process of treatment. TMAs excel at the development of new tumor markers that are prognostic. There is a debate about the utility of prognostic markers, where they forecast the behavior of a tumor, but are not useful in making a medical decision.

Nonetheless, TMAs are most commonly used in description of tumor behavior, either linked to outcome, or in some instances description of signaling pathways of growth and differentiation. The test of a marker for this utility is its capacity to add knowledge. Markers that provide information that is not greater than what can be determined by grade and stage are of little value (15).

Lastly are predictive markers: these are markers that can predict a response to a specific treatment, predict toxicity, or are used a decision point in clinical care. These biomarkers directly predict the individuaTs response to a set of conditions and are more commonly directed at drugable targets. Development of predictive markers is complex and multistep, and is central to the approach of personalized medicine.

Mechanistically, how investigators find themselves needing a TMA is even more complex. Routinely TMAs are used more as a validation tool than a discovery tool; however, they can be applied for raw discovery. The process of discovery with a TMA is to collect a series of markers and apply them to a TMA in an effort to discern some function. Typically this is accomplished by applying antibodies of markers that have been described in the literature as having a theoretic or predicted interaction, or the antibody reagent is new. Currently a widescale screening approach is underway with the human protein atlas, where antibodies to all predicted peptides are being generated and screened on TMAs of normal tissue and tumors (www.proteinatlas.org).

More commonly the approach for an investigator is to carry out some discovery method (described in the other chapters of this text) then seeking to confirm and generalize the finding. Although that appears to be a common path, different discovery approaches alter the nature of validation.

Most commonly expression microarrays have been the discovery platform that generate interest in the application of a TMA. The general advantage is that if immunohistochemistry is employed, the validation moves from transcriptional to proteomic $(17,18)$. The downside to this is twofold, (a) you have to have an antibody to the encoded protein and (b) the correspondence of transcriptional upregulation to upregulation at the protein level is limited. Simply put, for every target shown upregulated at the transcriptional level, flip a coin to 
predict its status at the protein. There are multiple levels of biologic control between transcription and the presence of protein in a cell, compounded with the nature of detection of the protein with immunohistochemistry. One advantage of the TMA approach is that it significantly extends the number of specimens examined. Expression array experiments are typically performed on limited numbers of samples (tens), while validation on a TMA will routinely involve hundreds of samples which can easily cover the spectrum of tumor grade and stage. In the event an antibody is not available, or cannot be logically developed, KNA in situ hybridization can be utilized to validate the expression array. As assays for mRNA have had limited clinical adoption, the approach of porting a discovery from the transcriptome to proteome has been more popular.

Increasingly proteomic methodologies are being applied to discovery of tumor biomarkers, and TMAs again are called on to validate results. For those methods that are not based on the use of antibodies, the approach and utility is similar to expression arrays: identification of an antibody against the target and validation against a larger cohort of tissue. In the instance where the original discovery methodology included the use of an affinity reagent, the tumor marker is already at the proteomic level. Here the success of validation seems improved, as the issues of transcriptome and proteome concordance are not at play (19). Most commonly antibody array and reverse-phase array discovery methods are supplying targets for the TMA analysis.

Lastly, there is the issue of where discovery begins and validation ends. Demonstration of a tumor marker having utility in a single cohort is not sufficient to see its introduction into clinical care (2). Most candidate tumor markers are first screened on TMAs without clinical information that allows simple determination of expression pattern and prevalence in a statistically relevant number of specimens. If they warrant further investigation, they are typically tested on a specific tumor panel for which clinical information (outcome) is available to determine utility If the tumor marker is a predictive marker, additional cohorts from clinical trials of patients who did and did not respond to the therapy are generally pursued. Lastly, and unfortunately not commonly enough, a tumor marker should be screened on population-based tumor TMAs that allow calculation of real prevalence rates for the marker and identification of populations with specific profiles (20).

\section{Building, Buying, or Collaborating on a TMA?}

Investigators have typically one of two reactions to planning to use a TMA: "I will build my own," or "I will buy one," but fail to consider collaboration. There are pros and cons of each, outlined as follows. Ultimately the answer should be at least two of the three options, as revalidation on a second independent cohort is an essential element of generalizing a novel finding.

Although construction ofaTMAisnot particularly challenging, obtaining the tissue is not simple, and optimally a histopathologist should review slides of the donor blocks before construction of the TMA. The instrumentation for array construction is becoming more common and more affordable; however, purchase of an arrayer is a poor investment if only one array or a few targets are to be explored. From an economic point of view, TMAs are 
best produced in high-volume environments where the appropriate team of researchers, pathologists, and technical staff can be assembled. This is not to dissuade construction of a TMA, but rather to temper the enthusiasm with the challenges.

Purchase of a TMA is simple: get on the web and go. Numerous vendors offer TMAs of a wide variety of tumors and normal tissues. You get what you paid for. TMAs with large numbers of specimens and extensive clinical information are very expensive. Vendors are only able to obtain a limited number of high-quaJity ( $>90 \%$ of cores present) sections and have cost associated with obtaining the material, annotation of outcome, and construction of the TMA. Other TMAs of good quality are generally available, but may lack clinical information. For some purposes, this is fine, and in other instances, will not address the question. Unfortunately there are too many cut-rate TMAs constructed of tissue obtain from undocumented sources. The phrase "junk in, junk out" best describes these TMAs. Tissue is fundamentally a reagent and must be of appropriate quality to yield valid results. In general commercial TMAs are a useful resource and provide a stepping stone in the process of confirming a finding, but fail to produce a strong test in a clinically relevant cohort of patients.

The best analogy of TMA slides is that they are like baseball trading cards. You do not need ten identical Mickey Mantles, and would happily trade a Mantle for a Hank Aaron, if you do not have one in your collection. Very few projects require all the TMA sections that are produced, and collaboration via sharing TMAs is an effective means of leveraging the expense and effort that goes into construction of a high-quality TMA. Currently there are no TMA "clearinghouses," but in general it is not difficult to identify a group that might have an appropriate TMA from the literature. Alternatively, if tissue is available, a researcher should investigate developing a collaboration with a TMA core lab to produce a new TMA. Often the TMA core lab will retain a block for their own use, or to develop other collaborations, and produce one or more for the collaborator who initiates the project and provides the tissue.

Mentioned previously was the issue of ethical approval to use human tissue in research, but equally important, and too frequently ignored are the contractual obligations of sharing research tissue that are an element of academic collaborations. Typically referred to as Material Transfer Agreements (MTAs) these are the contractual agreements that stipulate ownership, intellectual property rights, allowed uses, and agreements on publication of material obtained from collaboration. Many investigators tend to ignore them, at their own peril. Large multi-institutional collaborative TMAs are frequently delayed because of issues in obtaining the MTAs. In some instances one MTA is required for construction of the array, and a separate MTA will be utilized for distribution of the TMA slides.

\section{Have TMA, What Next?}

Once a researcher has the TMA and has chosen the assay to perform, the real work begins. TMAs have taught researchers more about assays and specimen quality than anticipated 18). It is very easy and common to discover a failure in the reagents and assays that was previously unanticipated. All assays on TMAs should be repeated in some fashion. Pilot 
arrays on sections of lower quality (fewer cores) are highly recommended as a means of fine tuning an assay and not wasting valuable resources.

Regardless of the assay, the real challenge is interpretation of the results and analysis of the data. TMAs are tissues and contain cytomorphologic and histomorphologic information. Although not all stains require analysis by a pathologist, consultation is highly recommended. It is not possible to a priori determine the means of categorizing the results of a TIMA before the staining has been done. For well-described tumor markers the literature is certainly a starting point. This certainly creates complexity in marrying results from some discovery platforms with TMA results (18).

Evaluation of immunohistochemistry relies on appreciation of location and intensity of staining within a specific cell population. In clinical use, this is most commonly simplified to interpretation as positive or negative, which has the advantages of simplified analysis and high reproducibility. However that is not the nature of science, where quantification is desired. Frequently two parameters - the percentage of cells expressing a marker and the intensity of the marker - are reported. When performed manually, the data are qualitative and often reported on scales of $0-3$ or 4 . When quantified with software, more quantitative (continuous scale) results are possible.

Ultimately it is not the raw data that is key, but the analysis (18). Analysis ofTMA results frequently confuses researchers, and well it should. Clinically immunohistochemistry is most robust as a positive/negative assay, and if a real clinical utility is envisioned, this must be kept in mind. Kaplan-Meier survival curves are typically generated on binary data (positive/negative) or a limited number of categories. At the other end of the spectrum, pathway analysis based on correlation, heat maps, and hazard proportion models are best carried out on quantitative data with dynamic range not adequately obtained with qualitative analysis. In the end, the analysis stands depends on the greatest amount of information that can be obtained from the combination of clinical parameters and characterization of the expression pattern.

\section{Conclusions}

The tissue microarray has become an indispensable tool in biomedical research. As the embodiment of high-throughput pathology and the means of translating tumor markers from discoveries to tools of clinical relevance they have advanced biomedical research significantly over the last decade. The TMA platform remains challenging because working with tissue remains challenging - at the level of collection, annotation, assay performance, and most importantly interpretation.

\section{References}

1. Kononen J, Bubendorf L, Kallioniemi A, Barlund M, Schraml P, Leighton S, T orhorst J, Mihatsch MJ, Sauter G, Kallioniemi OP. Tissue microarrays for high-throughput molecular profiling of tumor specimens. Nat Med. 1998 7;4(7):844-7. [PubMed: 9662379]

2. Braunschweig T, Chung JY, Hewitt SM. Tissue microarrays: bridging the gap between research and the clinic. Expert Rev Proteomics. 2005 6;2(3):325-36. [PubMed: 16000080] 
3. Becker D, Mihm MC, Hewitt SM, Sondak VK, Fountain JW, Thurin M. Markers and tissue resources for melanoma: meeting report. Cancer Res. 200611 15;66(22):10652-7. [PubMed: 17108101]

4. Khanna C, Wan X, Bose S, Cassaday R, Olomu O, Mendoza A, Yeung C, Gorlick R, Hewitt SM, Helman LJ. The membranecytoskeleton linker ezrin is necessary for osteosarcoma metastasis. Nat Med. 2004 2;10(2):182-6. [PubMed: 14704791]

5. Whiteford CC, Bilke S, Greer BT, Chen Q, Braunschweig TA, Cenacchi N, Wei JS, Smith MA, Houghton P, Morton C, Reynolds CP, Lock R, Gorlicb R, Khanna C, Thiele CJ, Takikita M, Catchpoole D, Hewitt SM, Khan J. Credentialing preclinical pediatric xenograft models using gene expression and tissue microarray analysis. Cancer Res. 2007 1 1;67(1):32-40. [PubMed: 17210681]

6. Hewitt SM. Design, construction, and use of tissue microarrays. Methods Mol Biol. 2004;264:6172. [PubMed: 15020780]

7. Schoenberg Fejzo M, Slamon DJ. Frozen tumor tissue microarray technology for analysis of tumor RNA, DNA, and proteins. Am J Pathol. 2001 11;159(5): 1645-50. [PubMed: 11696425]

8. Gillespie JW, Best CJ, Bichsel VE, Cole KA, Greenhut SF, Hewitt SM, Ahram M, Gathright YB, Merino MJ, Strausberg RL, Epstein JI, Hamilton SR, Gannot G, Baibakova GV, Calvert VS, Flaig MJ, Chuaqui RF, Herring JC, Pfeifer J, Petricoin EF, Linehan WM, Duray PH, Bova GS, EmmertBuck MR. Evaluation of non-formalin tissue fixation for molecular profiling studies. Am J Pathol. 2002 2;160(2):449-57. [PubMed: 11839565]

9. Goldstein NS, Hewitt SM, Taylor CR, Yaziji H, Hicks DG; Members of Ad-Hoc Committee On Immunohistochemistry Standardization. Recommendations for improved standardization of immunohistochemistry. Appl Immunohistochem Mol Morphol. 2007 6;15(2):124-33. [PubMed: 17525622]

10. Wang Y, Hewitt SM, Liu S, Zhou X, Zhu H, Zhou C, Zhang G, Quan L, Bai J, Xu N. Tissue microarray analysis of human FRAT1 expression and its correlation with the subcellular localisation of beta- catenin in ovarian tumours. BrJCancer. 20063 13;94(5):686-91.

11. Geiszt M, Lekstrom K, Brenner S, Hewitt SM, Dana R, Malech HL, Leto TL. NAD(P) H oxidase 1, a product of differentiated colon epithelial cells, can partially replace glycoprotein 91phox in the regulated production of superoxide by phagocytes. J Immunol. 20037 1;171(1):299-306. [PubMed: 12817011]

12. Huang HE, Chin SF, Ginestier C, Bardou VJ, Adelaide J, Iyer NG, Garcia MJ, Pole JC, Callagy GM, Hewitt SM, Gullick WJ, Jacquemier J, Caldas C, ChafFanet M, Bimbaum D, Edwards PA. A recurrent chromo-some breakpoint in breast cancer at the NRG1/ neuregulin 1/heregulin gene. Cancer Res. 200410 1;64(19):6840-4. [PubMed: 15466169]

13. Femandez DC, Bhargava R, Hewitt SM, Levin IW. Infrared spectroscopic imaging for histopathologic recognition. Nat Biotechnol. 2005 4;23(4):469-74. [PubMed: 15793574]

14. Takikita M, Chung JY, Hewitt SM. Tissue microarrays enabling high-throughput molecular pathology. Curr Opin Biotechnol. 2007 8;18(4):318-25. [PubMed: 17643281]

15. Hewitt SM, Takikita M, Braunschweig T, and Chung J-Y. (2007) Predictive tissue biomarkers: The promise \& the challenge. Biomarkers Med. 1(2): 313-318.

16. Nishizuka S, Chen ST, Gwadry FG, Alexander J, Major SM, Scherf U, Remhold WC, Waltham M, Charboneau L, Young L, Bussey KJ, Kim S, Lababidi S, Lee JK, Pittaluga S, Scudiero DA, Sausville EA, Munson PJ, Petricoin EF 3rd, Liotta LA, Hewitt SM, Raffeld M, Weinstein JN. Diagnostic markers that distinguish colon and ovarian adenocarcinomas: identification by genomic, proteomic, and tissue array profiling. Cancer Res. 2003 1;63(17):5243-50. [PubMed: 14500354]

17. Chuaqui RF, Bonner RF, Best CJ, Gillespie JW, Flaig MJ, Hewitt SM, Phillips JL, Krizman DB, Tangrea MA, Ahram M, Linehan WM, Knezevic V, Emmert-Buck MR. Postanalysis follow-up and validation of microarray experiments. Nat Genet. 2002 12;32 Suppl:509-14. [PubMed: 12454646]

18. Hewitt SM. The application of tissue microarrays in the validation of microarray results. Methods Enzymol. 2006;410:400-15. [PubMed: 16938563] 
19. Braunschweig T, Kaserer K, Chung J-Y,Bilke S, Krizman D, Knezevic V, Hewitt SM. (2007) Proteomic expression profiling of thyroid neoplasms with antibody arrays. Proteomics Clin Appl. 1:264-271. [PubMed: 21136677]

20. Goodman MT, Hernandez BY, Hewitt S, Lynch CF, Cote TR, Frierson HF Jr, Moskaluk CA, Killeen JL, Cozen W, Key CR, Clegg L,Reichman M, Hankey BF, Edwards B. Tissues from population-based cancer registries: a novel approach to increasing research potential. Hum Pathol. 2005 7; 36(7):812-20. [PubMed: 16084952] 